EPJ Web of Conferences 43, 05009 (2013)

DOI: $10.1051 /$ epjconf/20134305009

(C) Owned by the authors, published by EDP Sciences, 2013

\title{
Asteroseismology of pulsating DA white dwarfs with fully evolutionary models
}

\author{
A.D. Romero ${ }^{\mathrm{a}}$, A.H. Córsico, L.G. Althaus and M.M. Miller Bertolami \\ Facultad de Ciencias Astronómicas y Geofísicas, Universidad Nacional de La Plata, \\ Argentina
}

\begin{abstract}
We present a new approach for asteroseismology of DA white dwarfs that consists in the employment of a large set of non-static, physically sound, fully evolutionary models representative of these stars. We already have applied this approach with success to pulsating PG1159 stars (GW Vir variables). Our white dwarf models, which cover a wide range of stellar masses, effective temperatures, and envelope thicknesses, are the result of fully evolutionary computations that take into account the complete history of the progenitor stars from the ZAMS. In particular, the models are characterized by self-consistent chemical structures from the centre to the surface, a crucial aspect of white dwarf asteroseismology. We apply this approach to an ensemble of 44 bright DAV (ZZ Ceti) stars.
\end{abstract}

\section{INTRODUCTION}

White dwarf asteroseismology is a powerful tool that fully exploits the comparison between the observed pulsation periods in white dwarfs and the periods computed for appropriate theoretical models. It allows us to infer details of the origin, internal structure and evolution of white dwarfs ([1][2][3]). ZZ Ceti (or DAV) stars constitute the most numerous group of degenerate variable stars. They are otherwise normal DA (H-rich atmospheres) white dwarfs that exhibit g(gravity)-mode pulsations. Recently, our group la Plata Stellar Evolution and Pulsation Research Group has performed for the first time a detailed asteroseismological study on an ensemble of 44 bright ZZ Ceti stars by employing fully evolutionary (that is, non static) DA white dwarf models $[4]^{1}$. Our asteroseismological approach basically consists in the employment of a large suite of detailed stellar models characterized by very accurate and updated physical ingredients. These models were produced by computing the complete evolution of the progenitor stars. We have applied successfully this approach to the hot GW Vir (or DOV) stars in the past (see [5], and references therein), and also recently to the V777 Her (DBV) star KIC 8626021, the first pulsating white dwarf discovered in the Kepler mission field [6].

\section{ENSEMBLE ASTEROSEISMOLOGY OF ZZ CETI STARS}

We have performed an asteroseismological analysis of 44 bright ZZ Ceti stars based on our new set of fully evolutionary DA white dwarf models. One of our targets is the archetypal ZZ Ceti star

\footnotetext{
a e-mail: aromero@fcaglp.unlp.edu.ar

${ }^{1}$ A complete database with tabulations of chemical profiles, pulsation periods and other quantities of interest can be freely downloaded from our website (http: //www. f caglp.unlp.edu.ar/evolgroup).

This is an Open Access article distributed under the terms of the Creative Commons Attribution License 2.0, which permits unrestricted use, distribution, and reproduction in any medium, provided the original work is properly cited.
} 


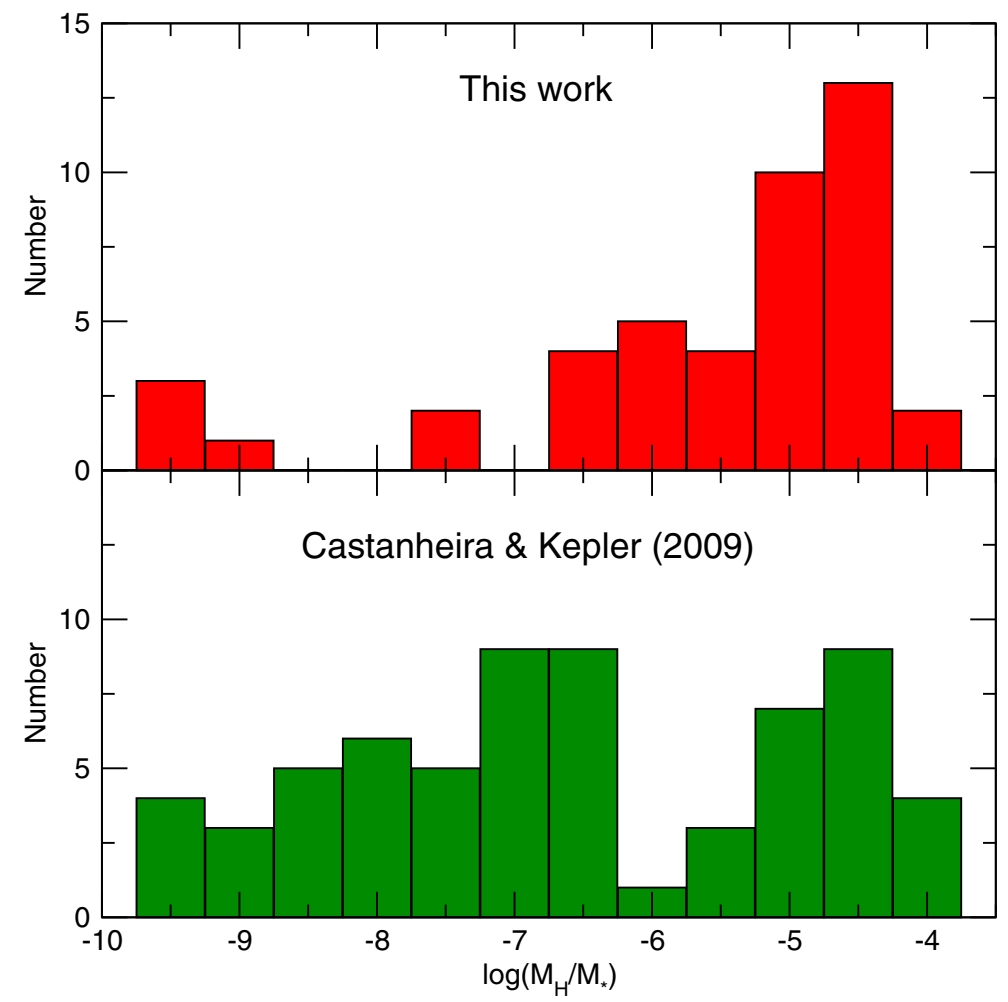

Figure 1. Histograms showing the $\mathrm{H}$ envelope thickness distribution for the sample of 44 bright $\mathrm{ZZ}$ Ceti stars (upper panel), and the results of [8] for a sample of 83 DAV stars (lower panel).

G117-B15A, for which we obtain a single solution regarding the thickness of the H layer, thus removing the longstanding ambiguity reported in previous works [5]. For the asteroseismological analysis of the ensemble of $44 \mathrm{ZZ}$ Ceti stars, our main results are:

- The mean value of the asteroseismological mass $\left(0.636 \pm 0.019 M_{\odot}\right)$ is in excellent agreement with our mean spectroscopic mass $\left(0.630 \pm 0.028 M_{\odot}\right)$, and in line with the mean mass of DA white dwarfs inferred by [7] $\left(0.647 M_{\odot}\right)$.

- There exists a range of thicknesses of the $\mathrm{H}$ envelope in the studied ZZ Ceti stars, in qualitative agreement with the results of [8]. Our distribution has a strong peak at thick envelopes $\left[\log \left(M_{\mathrm{H}} / M_{*}\right) \sim-4.5\right]$ and another much less pronounced peak at very thin envelopes $\left[\log \left(M_{\mathrm{H}} / M_{*}\right) \sim-9.5\right]$ (see Fig. 1). This result supports the belief that white dwarfs in the solar neighborhood harbor a broad range of hydrogen-layer thickness.

\section{References}

[1] D.E. Winget, S.O. Kepler, ARA\&A 46, 157 (2008), 0806.2573

[2] G. Fontaine, P. Brassard, PASP 120, 1043 (2008)

[3] L.G. Althaus, A.H. Córsico, J. Isern, E. García-Berro, A\&A Rev. 18, 471 (2010), 1007.2659

[4] A.D. Romero, A.H. Córsico, L.G. Althaus, S.O. Kepler, B.G. Castanheira, M.M. Miller Bertolami, MNRAS 420, 1462 (2012), 1109.6682 
Ageing Low Mass Stars: From Red Giants to White Dwarfs

[5] A.H. Córsico, L.G. Althaus, M.M. Miller Bertolami, E. García-Berro, A\&A 499, 257 (2009), 0903.3628

[6] A.H. Córsico, L.G. Althaus, M.M. Miller Bertolami, A. Bischoff-Kim, A\&A 541, A42 (2012), 1112.5882

[7] R.E. Falcon, D.E. Winget, M.H. Montgomery, K.A. Williams, ApJ 712, 585 (2010), 1002 . 2009

[8] B.G. Castanheira, S.O. Kepler, MNRAS 396, 1709 (2009) 